\title{
Effects of temperature on surface clusters by molecular dynamics simulation
}

\author{
Sheng-Rui Jian ${ }^{\mathrm{a}}$, Te-Hua Fang ${ }^{\mathrm{b}, *}$, Der-San Chuu ${ }^{\mathrm{a}}$ \\ ${ }^{a}$ Institute and Department of Electrophysics, National Chiao Tung University, Hsinchu 300, Taiwan \\ ${ }^{\mathrm{b}}$ Department of Mechanical Engineering, Southern Taiwan University of Technology, Tainan 710, Taiwan \\ Received 26 December 2002; received in revised form 10 March 2003; accepted 10 March 2003
}

\begin{abstract}
This article discusses the physical mechanisms for the evaporation phenomena of argon clusters on surfaces under various temperatures with the aid of molecular dynamics analysis by means of the Stoddard-Ford potential. Our simulated results indicate that the evaporation rate of the argon clusters increased drastically when the temperature was increased but the contact angle decreased. Furthermore, the thermal stability of the argon clusters is also discussed here. The evaporation mechanisms of argon clusters are clearly shown with the aid of molecular dynamics.
\end{abstract}

(C) 2003 Elsevier Science B.V. All rights reserved.

PACS: 68.03.Fg; 61.46. +w; 61.25.Bi

Keywords: Molecular dynamics; Stoddard-Ford potential; Clusters; Evaporation

\section{Introduction}

Nanoclusters are currently attracting considerable attentions for their use in lithography, deposition, electronic devices, etc. because of their unique characterizations [1-3]. Better investigation of the mechanisms associated with nanometerscale clusters has become increasingly important in the field for both theoretical and experimental research $[4,5]$.

The technique is rapidly developing, yet it is still quite difficult to describe the phenomena on an atomic scale. Accordingly, molecular dynamics provides useful information for understanding

\footnotetext{
*Corresponding author. Fax: + 886-62-42-5092.

E-mail address: fang@mail.stut.edu.tw (T.-H. Fang).
}

atomic behaviors that are difficult to obtain experimentally. All physical phenomena, surface tension, evaporation, condensation and so on come from the intermolecular interaction.

It is well known that molecular dynamics simulations, by virtue of their high temporal and spatial resolutions, offer novel insights into atomistic mechanisms. Recently, a number of researchers have used atomistic simulations to investigate the physical mechanisms of clusters [610], but there still are many infinitesimal details that are not clearly understood. Therefore, the emphasis of this study is focused on the evolution of nanometer-size argon surface clusters in the course of the evaporation mechanisms at various temperatures via molecular dynamics simulation technique. 
During the simulation processes, it was found that the "vacancy" event and the influence of temperature played an important role in this system.

\section{Molecular dynamics methodology}

The evaporation of argon clusters on surfaces was investigated using molecular dynamics simulations. Periodic boundary conditions (PBC) [11] are used in the transverse directions ( $x$ - and $y$ axis), and the bottom three layers of the Pt atoms of the substrate are fixed in space [12]. The PBC is also used for the liquid argon nanoclusters. The force acting on an individual atom is obtained by summing the forces contributed by the surrounding atoms; meaning that, the atoms are described to interact via the Stoddard-Ford potential [13], as follows:

$$
\begin{aligned}
\phi\left(r_{i j}\right)= & 4 \varepsilon\left\{\left[\left(\frac{\sigma}{r_{i j}}\right)^{12}-\left(\frac{\sigma}{r_{i j}}\right)^{6}\right]+\left(\frac{r}{r_{\mathrm{cut}}}\right)^{2}\right. \\
& \times\left[6\left(\frac{\sigma}{r_{\mathrm{cut}}}\right)^{12}-3\left(\frac{\sigma}{r_{\mathrm{cut}}}\right)^{6}\right] \\
& \left.-7\left[\left(\frac{\sigma}{r_{\mathrm{cut}}}\right)^{12}-4\left(\frac{\sigma}{r_{\mathrm{cut}}}\right)^{6}\right]\right\},
\end{aligned}
$$

where $\phi\left(r_{i j}\right)$ is a pair energy function, $r_{\text {cut }}$ (cut-off distance) is set at $3.5 \sigma, \sigma=3.40 \times 10^{-10} \mathrm{~m}$ and $\varepsilon=$ $1.67 \times 10^{-21} \mathrm{~J}$. The Stoddard-Ford potential has been selected for these simulations and it has been used previously in another study [14].

The force on the $i$ th atom resulting from the interaction of all the other atoms can be derived from Eq. (1) such that

$F_{i}=-\sum_{j=1, j \neq i}^{N} \nabla_{i} \phi\left(r_{i j}\right)=N m_{i} \frac{\mathrm{d}^{2} r_{i}(t)}{\mathrm{d} t^{2}}$,

where $F_{i}, m_{i}, r_{i}$ and $N$ are denoted as: the resultant force on the $i$ th atom, the mass of $i$ th atom, the position of the $i$ th atom and the total number of atoms, respectively.

The initial configuration of the substrate was a face-centered cubic (FCC) lattice. Initial velocities were assigned from the Maxwell distribution [11] and the magnitudes were adjusted with the purpose of keeping the temperature in the system constant according to

$v_{i}^{\text {new }}=v_{i}^{\text {old }}\left\{\frac{N_{\mathrm{f}} k_{\mathrm{B}} T_{0} N}{2}\left[\sum_{i=1}^{N} \frac{m_{i}\left(v_{i}^{\text {old }}\right)^{2}}{2}\right]^{-1}\right\}^{1 / 2}$,

where $v_{i}$ is the velocity of $i$ th atom, $T_{0}$ is a specified temperature, $k_{\mathrm{B}}=1.381 \times 10^{-23} \mathrm{~J} \mathrm{~K}^{-1}$ is the Boltzmann's constant and $N_{\mathrm{f}}$ is the freedom of the system. The initial displacement and velocity were values determined independently and the time integration of motion was performed by Gear's fifth predictor-corrector method [11] with time step of $1 \mathrm{fs}$.

The initial atomic configuration of the simulated system is illustrated in Fig. 1. The eight-layers of the FCC, each of which contained about 640 atoms, were set up as the substrate. An argon cluster of $3 \mathrm{~nm}$ in size (with a cluster consisting of 1372 atoms) was placed on the substrate. This was not claimed to be a truly minimum array but was rather an empirically derived practical array. For this simulation, the NVT model [15] was used to control the number of atoms $N$, volume $V$ and temperature $T$; in addition, the substrate was initially assumed to have a well-defined atomic surface and the atomic array model of the surface was constructed at a specific constant temperature. In addition, the velocities of the substrate atoms at a specific constant temperature were satisfied with the Maxwell velocity distribution.

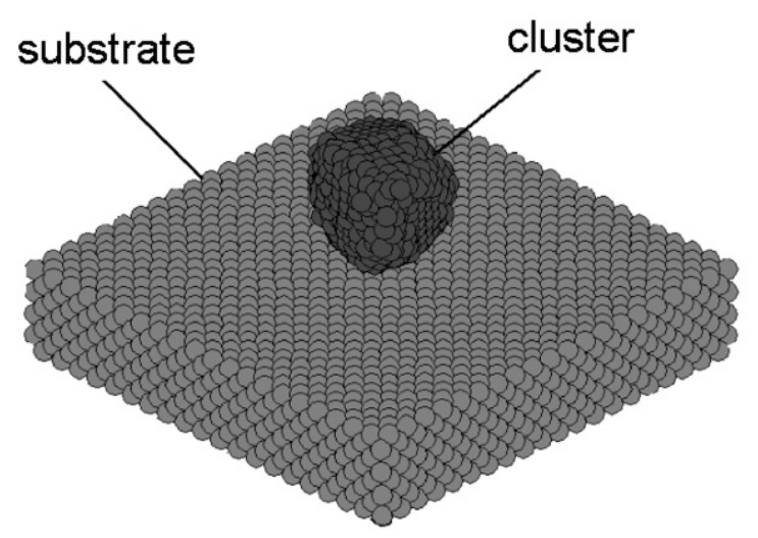

Fig. 1. Initial configuration of the simulated system using the molecular dynamics model. 


\section{Results and discussions}

Fig. 2 shows time step snapshots of the trajectory of argon clusters in which adsorption and evaporation phenomena can be observed. At the time steps of $120(\times 100 \mathrm{fs})$ to $500(\times 100 \mathrm{fs})$, it can be seen that the volume expansion of the vapor argon atoms occurs rapidly at three various temperatures. In addition to that, there are adsorption and evaporation phenomena taking place during the simulation processes and these phenomena are strongly dependent upon the temperature of the system.

It is important to note that at the highest temperature of $450 \mathrm{~K}$, the smaller argon clusters formed and moved away from the surface. As the evaporation process advanced, it becomes clear that a large amount of the vapor argon atoms have already moved far away from the surface and this is illustrated in Figs. 2(b), (c).
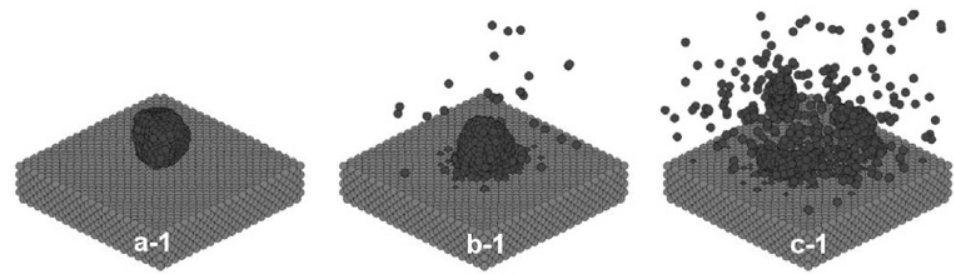

time step $=120(\times 100 f s)$
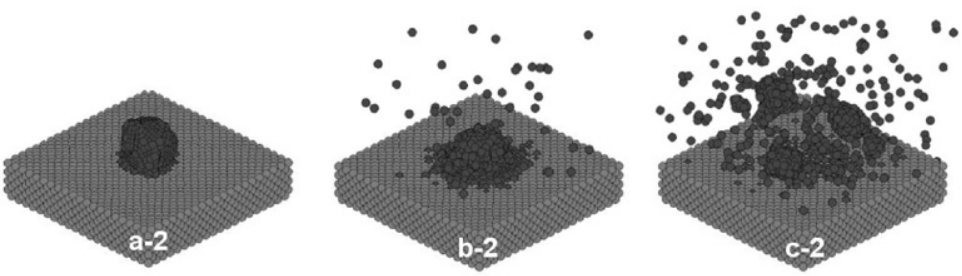

time step $=240(\times 100 f s)$
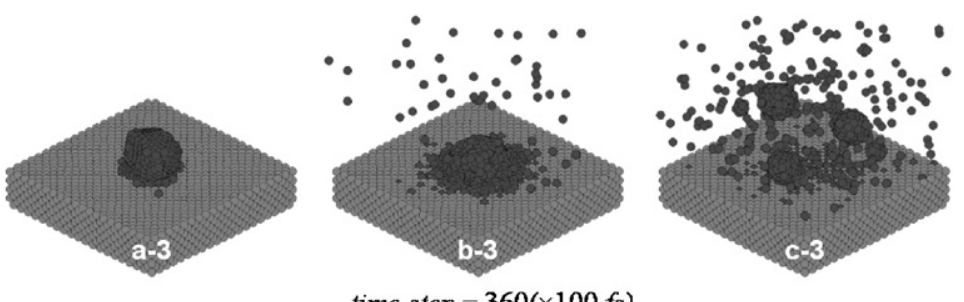

time step $=360(\times 100 f s)$
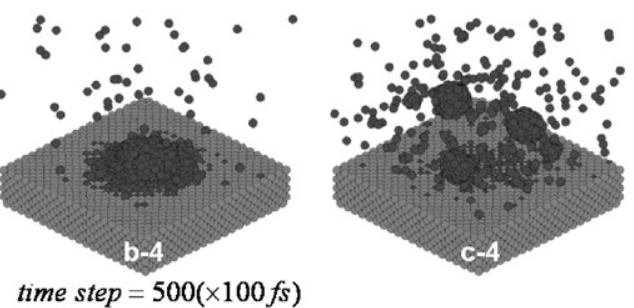

(a) $50 \mathrm{~K}$

(b) $250 \mathrm{~K}$

(c) $450 \mathrm{~K}$

Fig. 2. The adsorption and evaporation phenomena at various time steps and under various temperatures: (a) $50 \mathrm{~K}$, (b) $250 \mathrm{~K}$, and (c) $450 \mathrm{~K}$, respectively. 
The original cluster was separated into several smaller clusters because the system's temperature was raised, i.e. the energy of the system was
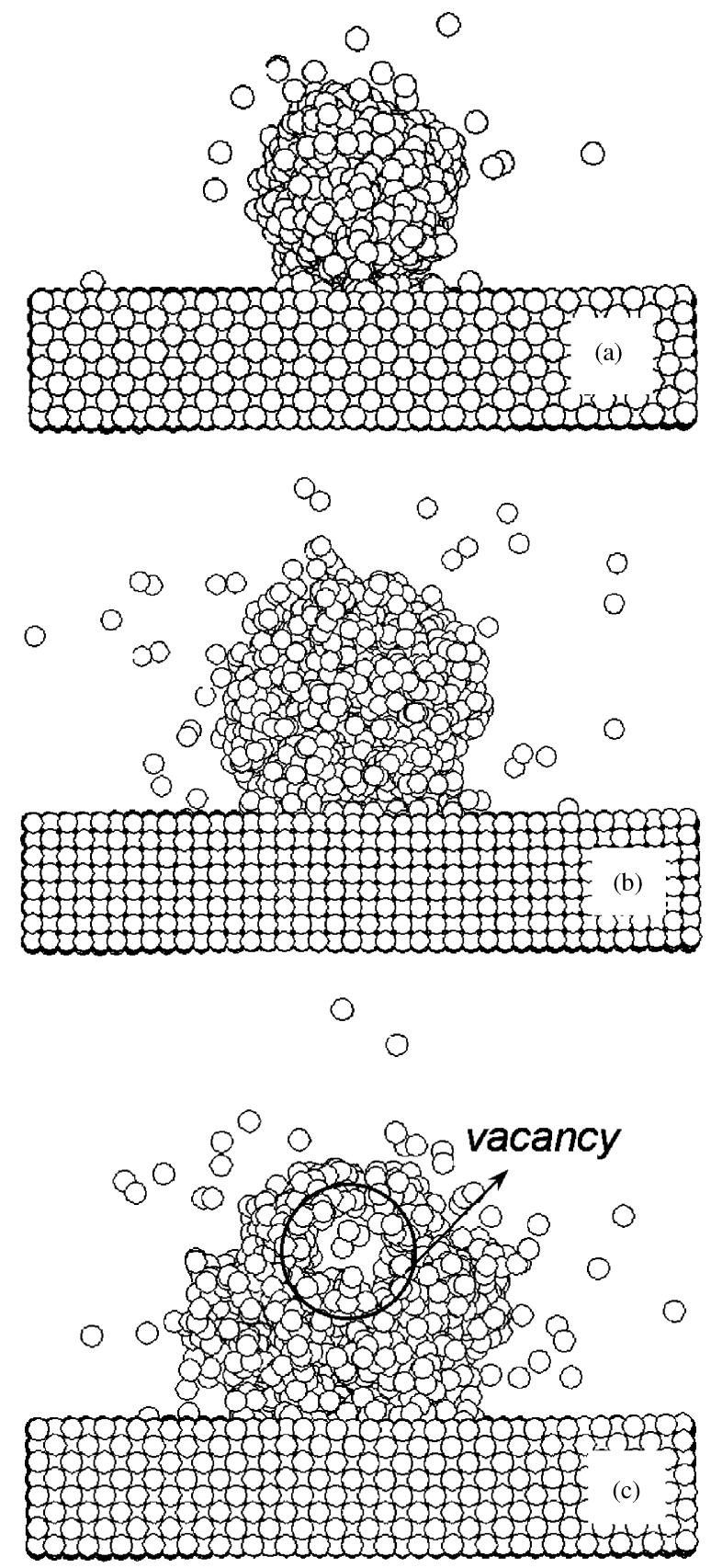

Fig. 3. Snapshots of the evaporating argon clusters configuration at a temperature of $250 \mathrm{~K}$ during the $120-240(\times 100 \mathrm{fs})$ time steps. increased to reach a sufficient level to break the atomic bonds (van der Waals forces) and then the evaporation phenomena began. During the simulation process, another new phenomenon was observed this was a "vacancy" materialized at the temperature of $250 \mathrm{~K}$ and is displayed in Fig. 3. The clusters showed violent variations because the energy being increased rapidly, which produced a "vacancy" or split into a number of smaller clusters and into weaker binding energy which allowed the atoms to escape from the surface.

Fig. 4 depicts the time step snapshots of the contact angle $(\theta)$. It can be seen that when the temperature is raised, the contact angle of the temperature at $250 \mathrm{~K}$ is smaller than the contact angle of the temperature at $150 \mathrm{~K}$. The contact angle experiments of Rowan et al. [16] and Hua et al. [17] provide further evidence of the evaporating phenomena of microscale clusters and the variation of the size of the clusters. The simulated results for the nanometer-scale clusters in this study were similar to those in previous studies. A linear relationship of the constant angle and the time steps were observed in angles between $50^{\circ}$ and $140^{\circ}$ and may be

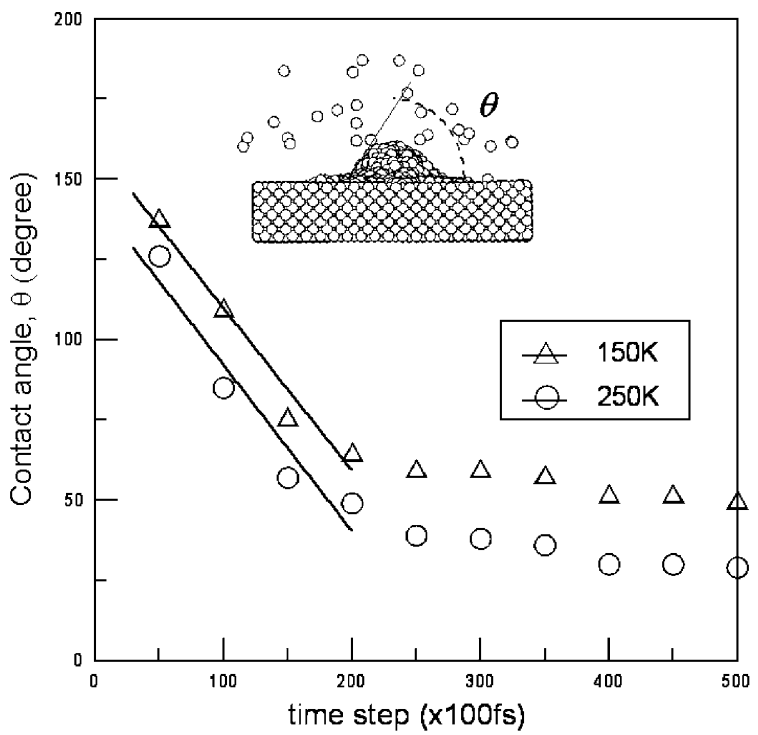

Fig. 4. The contact angle at various time steps for 150 and $250 \mathrm{~K}$, respectively. 
expressed as

$\theta=-A y+B$,

where $\theta$ is the contact angle, $y$ is the time step, where $A$ and $B$ are constant values obtained by curve fitting.

Fig. 5 shows the ratio of $N_{\mathrm{e}} / N_{\text {cluster }}$ increased as the time steps were increased. Simplification parameters representing the ratio of cluster evaporation were defined as $N_{\mathrm{e}} / N_{\text {cluster }}$, where $N_{\mathrm{e}}$ is the number of evaporating atoms of the cluster and $N_{\text {cluster }}$ is the total number of atoms of the cluster. The evaporating atoms increased rapidly at higher temperatures. The values of $N_{\mathrm{e}} / N_{\text {cluster }}(\%)$ ranged from $0.001 \%$ to $27.33 \%$ for temperatures of $50-450 \mathrm{~K}$. This is because the higher temperature of $450 \mathrm{~K}$ can cause the disintegration of the clusters. A more detailed disintegration of the clusters can also be seen in Fig. 2.

The evaporation ratio, i.e. $N_{\mathrm{e}} / N_{\text {cluster }}$ for formation of a vaporization region between $(200(\times 100 \mathrm{fs}))$ and $(400(\times 100 \mathrm{fs}))$ time steps, at the temperatures of 250,350 and $450 \mathrm{~K}$ ranged $3.72-5.03 \%, \quad 15.67-18.22 \%$ and $17.20-26.97 \%$, respectively. The simulated results deduced are in agreement with the evaporation behavior of other

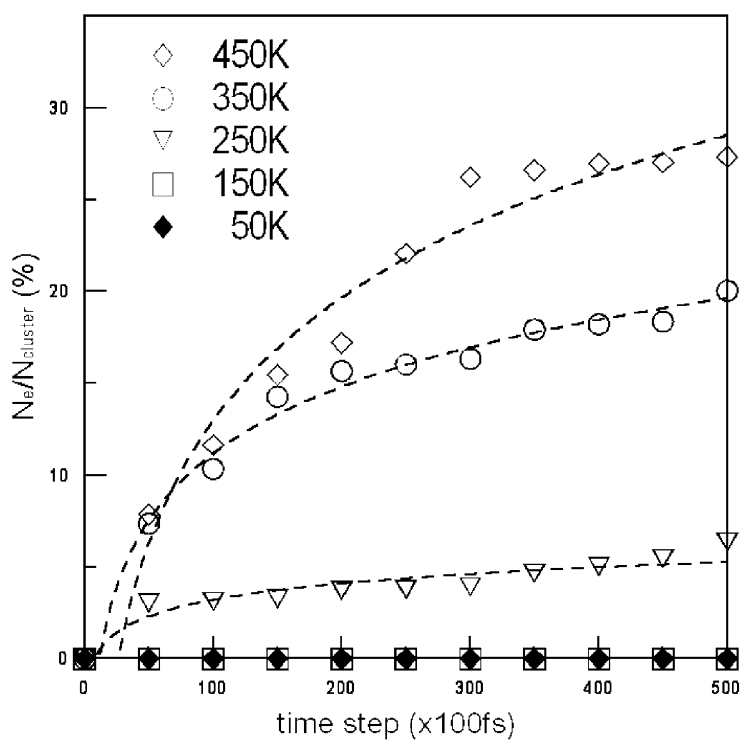

Fig. 5. The evaporation ratios at different time steps and temperatures. similar macroscopic experiments [18]. In addition to that, the expected rate of evaporation was $3.33 \times 10^{24}, 0.73 \times 10^{27}, 2.49 \times 10^{27}, 4.45 \times 10^{27}$ and $6.35 \times 10^{27}\left(1 \mathrm{~m}^{-2} \mathrm{~s}^{-1}\right)$ for the temperatures of $50,150,250,350$ and $450 \mathrm{~K}$, respectively. The evaporation rate is defined as the number of atoms evaporating per unit area of surface per unit time. These were based on previous studies [18] and are commonly used to calculate evaporation rate. In comparing the expected evaporation rates of 50,150 and $250 \mathrm{~K}$ to the calculated rates, it was discovered that the expected rates were higher than the calculated rates of $2.58 \times 10^{23}\left(1 \mathrm{~m}^{-2} \mathrm{~s}^{-1}\right)$, $5.15 \times 10^{23}\left(1 \mathrm{~m}^{-2} \mathrm{~s}^{-1}\right)$ and $2.25 \times 10^{27}\left(1 \mathrm{~m}^{-2} \mathrm{~s}^{-1}\right)$, respectively. At 350 and $450 \mathrm{~K}$, the expected rates were lower than the calculated rates of $7.08 \times$ $10^{27}\left(1 \mathrm{~m}^{-2} \mathrm{~s}^{-1}\right)$ and $9.66 \times 10^{27}\left(1 \mathrm{~m}^{-2} \mathrm{~s}^{-1}\right)$.

The calculated potential energy of the system at different time steps is shown in Fig. 6 and are plotted in exponential-like decay curves. In Fig. 6, it can be seen that the curve began to decrease abruptly at around $50(\times 100 \mathrm{fs})$. The general decaying behavior of the energy was caused by the attractive interatomic forces of the clusters.

From the kinetic theory of gases [19], the temperature was directly related to the kinetic

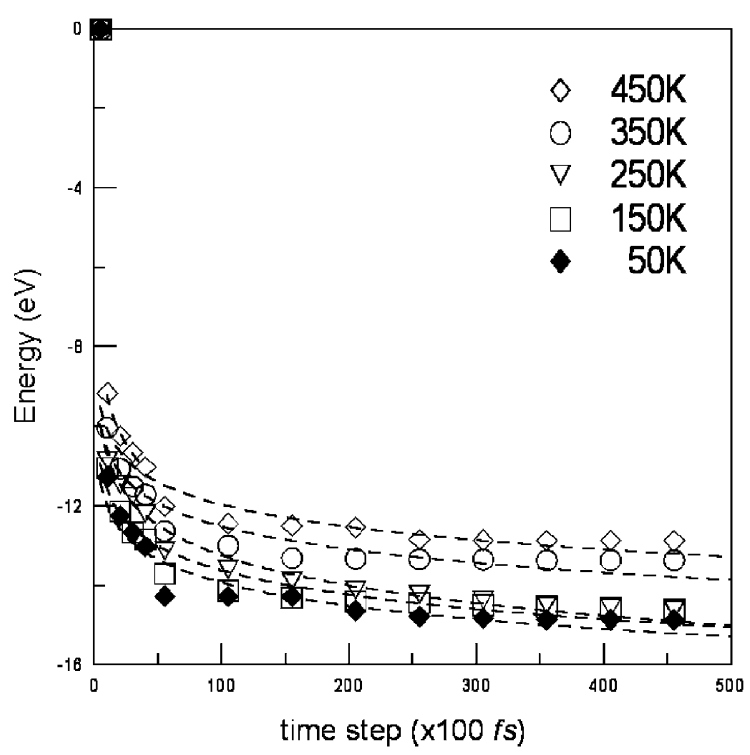

Fig. 6. The potential energy at various time steps and temperatures. 
energy. Thus, as the temperature was increased, the number of evaporating atoms increased and raised the potential energy of the system as expected. After complete evaporation, the system stabilized and the estimated energy in the higher temperature of $450 \mathrm{~K}$ (about $-12.87 \mathrm{eV}$ ) was larger than lower temperature of $50 \mathrm{~K}$ (about $-14.86 \mathrm{eV}$ ).

\section{Conclusion}

This study adopted the method of molecular dynamics to investigate the evaporation phenomena of nanometer-scale argon clusters on surfaces under various temperatures. A higher-temperature state manifests the formation of small clusters and the "vacancy" event. The results indicated that the probability of the number of vapor argon atoms increased and the evaporation ratios were evaluated to have been approximately 3.06-27.33\% depending on the temperature, but the contact angle decreased during evaporation. Summarizing, it was further confirmed that the equilibrium state of the argon clusters at various temperatures can provide qualitative results and the entire evaporation process can be successfully simulated by molecular dynamics.

\section{Acknowledgements}

This work was partially supported by the National Science Council of Taiwan, under Grant Nos. NSC91-2218-E218-001 and NSC91-2212-E218-007.

\section{References}

[1] F. Iacona, D. Pacitifici, A. Irrera, M. Miritello, G. Franzo, F. Priolo, D. Sanfilippo, S.G. Di, P.G. Fallica, Appl. Phys. Lett. 81 (2002) 3242.

[2] Y. Qiang, R.F. Sabiryanov, S.S. Jaswal, Y. Liu, H. Haberland, D.J. Sellmyer, Phys. Rev. B 66 (2002) 4404.

[3] M. Zheng, M. Yu, Y. Lin, R. Skomski, S.H. Lion, D.J. Sellmyer, V.N. Petryakov, Y.K. Verevkin, N.I. Polushkin, Salashchenko, Appl. Phys. Lett. 79 (2001) 2606.

[4] G. Scoles, The Chemical Physics of Atomic and Molecular Clusters, North-Holland, Amsterdam, 1990.

[5] H. Haberland, Clusters of Atoms and Moleculars, Springer, Berlin, 1994.

[6] J.H. Walther, P. Koumoutsakos, J. Heat Transfer 123 (2001) 741

[7] A. Rytkönen, S. Valkealahti, M. Manninen, J. Chem. Phys. 106 (1997) 1888.

[8] N. Yamaguchi, Y. Sasajima, K. Terashima, T. Yoshida, Thin Solid Films 345 (1999) 34.

[9] K. Yasuoka, M. Matsumoto, Y. Kataoka, J. Chem. Phys. 101 (1994) 7904.

[10] M.S. Korlie, Comput. Math. Appl. 39 (2000) 43.

[11] J.M. Haile, Molecular Dynamics Simulation: Elementary Method, Wiley, New York, 1992.

[12] Y. Isono, T. Tanaka, JSME Int. J. Ser. A: Mech. Mater. Eng. 40 (1997) 211.

[13] S.D. Stoddard, P.J. Ford, Phys. Rev. A 8 (1973) 1504.

[14] P. Yi, D. Poulikakos, J. Walther, G. Yadigaroglu, Int. J. Heat Mass Transfer 45 (2002) 2087.

[15] W.G. Hoover, Phys. Rev. A 31 (1985) 1695.

[16] S.M. Rowan, G. McHale, M.I. Newton, M. Toorneman, J. Phys. Chem. B 101 (1997) 1265.

[17] H. Hua, G.L. Ronald, J. Phys. Chem. B 106 (2002) 1334.

[18] A.J. Walton, Three Phases of Matter, Clarendon Press, Oxford, 1983.

[19] R.P. Feynman, R.B. Leighton, M. Sands, in: The Feynman Lectures on Physics, Vol. 1, Addison-Wesley, Reading, MA, 1963. 